\title{
Accuracy of subjectlve judgments of information in long-term memory
}

FRANK DAPOLITO, HOWARD GUTTENPLAN, and HAROLD STEINITZ, The University of Rochester, Rochester, N.Y. 14627

Thirty $S$ s received a general information test. A four-stage paradigm referred to as recall-judgmentelimination-choice was used to test $S$ 's ability to predict choice-recognition performance following incorrect recall. The results indicated that Ss can accurately predict which of the failed items will be subsequently recognized. These results were largely due to $S$ 's ability to reject or eliminate incorrect alternatives.

Following recall failure, additional testing procedures some times exhibit relatively complete retention of the failed item. For example, continued attempts at total or partial recall may eventually result in success (Brown \& McNeil, 1966). More commonly, correct recognition without additional training often follows recall failure (Postman, Jenkins, \& Postman, 1948). It is clear that the presence or absence of information in memory is not always accurately mirrored by recall performance.

The inability to recall an item of information is usually accompanied by "feelings-of-knowing." Hart $(1965,1966)$ has made use of the common discrepancy between recall and recognition measures to investigate whether the "feelings-ofknowing" which accompany recall failure might be used to predict subsequent recognition performance.

The accuracy of such predictions is important since S's judgements might be used in conjunction with recall tests to obtain more accurate indicators of retention. In general, Hart's results suggest that $S s$ are quite accurate in predicting which of the failed items will be correctly recognized.

The present study, which is a modification of Hart's (1965) experiment, employs a five-choice recognition test with more homogeneous alternatives. Also, an elimination procedure was added in an attempt to evaluate S's guessing strategies on the final recognition test.

\section{METHOD}

The Ss were 30 undergraduate students and each was tested individually. Fifty general information questions were read to $S$ at a rate of approximately three questions per minute. The questions were selected from the fields of literature, art, history, science, music, politics, geography, and sports. Except for book titles, it was possible for $S$ to answer each question with a single-word or double-word response. A sample question is: "Who is the author of The Three Musketeers?"

A four-stage procedure referred to as "recall-judgmentelimination-choice" was employed. In the initial stage Ss attempted to recall answers to each of the 50 questions. A forced-guessing procedure was used to insure a response to each question. The second stage was concerned with items S recalled (or guessed) incorrectly. Immediately following each failed item, S was asked to judge ("yes" or "no") whether or not he could recognize the correct answer from among five choices. All 50 questions were completed in this manner. In Stage 3, the items missed on the prior recall test were presented in written form on a $5 \times 8$ card with five alternatives typed below the question. The alternatives were chosen so as to be as "similar" to the correct choice as possible. For example, when the capital of a state was requested the five choices were the five major cities of the state and if the author of a particular book was asked for, the choices were authors of the same nationality. For the sample question mentioned earlier, the altematives were (a) Jean Racine, (b) Charles Baudelaire, (c) Michel Montaigne, (d) Jean Moliere, (e) Alexandre Dumas. The correct alternative occured at each position with approximately equal frequency. During this stage, $S$ was instructed to pronounce the alternatives which he definitely believed to be incorrect answers. Instructions stressed that $E$ wished to know which of the choices $S$ would not really consider in making his final decision. Following this $S$ was to choose the "correct" answer from the remaining alternatives. The recognition test trials were unpaced and all responses were recorded by $\mathrm{E}$.

\section{RESULTS AND DISCUSSION}

The proportion of questions correct on the initial recall test was .36. Of the questions failed on recall, . 46 were correctly recognized on the final recognition test. Thus, recognition performance following recall failure appeared to be well above the a priori chance level of .20 . It should be noted that during the judgment phase, the proportions of failed items receiving "yes" and "no" judgments were about equal, being .54 and .46 , respectively.

The principal question of interest was whether the proportions of items recognized would be significantly different for items receiving "yes" and "no" judgments. If S's judgments are independent of any stored information, then the proportion of correct recognitions given a "yes" judgment (C:yes) and the proportion correct given a "no" judgment (C:no) should be equal. At the opposite extreme, if $S$ can perfectly detect the presence of information which is unavailable through recall processes, then (C:yes) should approach unity and ( $\mathrm{C}:$ no) should be essentially chance performance on a five-choice test (assuming random selection from among the alternatives).

Table 1 presents the proportions (C:yes) and (C:no) for each of the $30 \mathrm{Ss}$. Pooling over all items and Ss, the proportion (C:yes) was .58 while the proportion (C:no) was .32. A t test for correlated proportions indicated that (C:yes) scores were significantly greater than (C:no) scores $[t(29)=6.52$, $\mathrm{p}<.001]$.

These results on recognition fully support those of Hart $(1965,1966)$ that $S s$ can reliably predict subsequent recognition performance after recall failure. However, it is clear that the proportion of recognitions following a "yes" judgment (.58) is considerably below unity which suggests that Ss' judgments are less than perfect.

Table 1

Proportions of Correct Recognitions Given "Yes" (c:yes) and "No" (c:no) Judgments for Each S

\begin{tabular}{cccccc}
\hline Subject & (c:yes) & (c:no) & Subject & (c:yes) & (c:no) \\
\hline 1 & .52 & .40 & 16 & .80 & .33 \\
2 & .46 & .22 & 17 & .60 & .31 \\
3 & .61 & .33 & 18 & .84 & .00 \\
4 & .60 & .50 & 19 & .74 & .00 \\
5 & .63 & .37 & 20 & .46 & .44 \\
6 & .47 & .33 & 21 & .58 & .29 \\
7 & .33 & .30 & 22 & .74 & .36 \\
8 & .75 & .25 & 23 & .64 & .43 \\
9 & .69 & .67 & 24 & .45 & .31 \\
10 & .65 & .16 & 25 & .70 & .19 \\
11 & .46 & .16 & 26 & .60 & .43 \\
12 & .59 & .57 & 27 & .40 & .50 \\
13 & .58 & .27 & 28 & .46 & .22 \\
14 & .46 & .56 & 29 & .58 & .20 \\
15 & .55 & .33 & 30 & .56 & .24 \\
\hline
\end{tabular}


The elimination procedure provided additional data on $\mathbf{S}$ ' capacity to detect stored but unavailable information. If S's judgments are based upon his ability to detect the amounts of partial information retained, then the differential information which exists should enable more efficient rejection of incorrect alternatives for items receiving "yes" judgments as well as superior recognition of correct answers. Moreover, if S detects the presence or absence of specific information, one might expect that proportion of cases where $S$ actually eliminates the correct alternative to be small for items receiving "yes" judgments.

For each $S$, the proportion of incorrect alternatives eliminated was obtained for items receiving "yes" and "no" judgments and a t test for correlated proportions was performed on these scores. As expected, Ss eliminated more incorrect alternatives following a "yes" judgment than following a "no" judgment $[\mathrm{t}(29)=5.18, \mathrm{p}<.001]$. Pooling over all items and Ss, the proportion of wrong answers eliminated was .58 for "yes" items and .29 for "no" items. Apparently the differential elimination of incorrect alternatives is one basis for Ss' superior performance on "yes" items.

The proportion of cases in which the correct alternative was actually eliminated was identical (.18) for items receiving "yes" and "no" judgments. This suggests the possibility that Ss are better at eliminating incorrect alternatives than at recognizing the correct response. However, this interpretation should remain provisional until more substantial data are available.

It seems reasonable to view choice-recognition behavior in terms of a sequential scanning process whereby $\mathrm{S}$ scans the alternatives, one at a time, and compares each alternative against a partial "trace." Perhaps S decides to eliminate an alternative only when there is a mismatch against the partial information retained. When there is no discrepancy $S$ keeps the alternative as a possible choice. Finally, $S$ chooses at random (approximately) from among the remaining alternatives. On this view, there should be differential elimination for "yes" and "no" items but it is not yet clear under what conditions the elimination of correct alternatives should be independent of S's judgment. Further research using additional testing procedures is needed to clarify the bases for S's "judgements-of-knowledge."

\section{REFERENCES}

BROWN, R., \& McNEILL, D. The "tip of the tongue" phenomenon Journal of Verbal Learning \& Verbal Behavior, 1966, 5, 325-337.

HART, J. T. Memory and the feeling-of-knowing experience. Journal of Educational Psychology, 1965, 56, 208-216.

HART, J. T. A methodological note on the feeling-of-knowing experiments. Journal of Educational Psychology, 1966, 57, 347-349.

POSTMAN, L., JENKINS, W. G., \& POSTMAN, D. L. An experimental comparison of active recall and recognition. American Journal of Psychology, 1948, 61, 511-519. 